\title{
A gradient theory based on the Aifantis theory using the Gurtin-Anand strain gradient plasticity approach
}

${ }^{1}$ Mathematics Unit, Distance Learning Institute, University of Lagos, Lagos, Nigeria, E-mail: aborokini@unilag.edu.ng

\begin{abstract}
:
This article investigates the differences between the Aifantis and Gurtin-Anand strain gradient plasticity. The fact that Gurtin-Anand strain gradient plasticity is richer than the Aifantis strain gradient plasticity provides a basis for which we could derive equivalent Aifantis flow rule and extend it to accommodate scalar dissipative hardening. It was found that the major difference between the Gurtin-Anand and Aifantis theories lies on the use of the codirectionality hypothesis and constraint imposed on the gradient of the flow direction.
\end{abstract}

Keywords: Aifantis theory, flow rule, Gurtin-Anand theory, strain-gradient plasticity

MSC 2010: 74C10

DOI: $10.1515 / \mathrm{jmbm}-2018-0012$

\section{Introduction}

Strain gradient plasticity deals with the study of the effects of plastic strain gradients on plastically deformed bodies at varying length scales. The subject of strain gradient plasticity has become a considerable aspect of plasticity with renewed interest in its theories and applications. Aifantis [1], [2] is known as a pioneering author on the mathematical study of strain gradient plasticity. He introduced into the classical theory of plasticity - through the yield criterion -terms relating to the Laplacian of the accumulation of plastic strain. This generalization which we shall refer to as the Aifantis theory, enables the determination of certain effects not captured by the conventional plasticity theory. For instance, the review done by Hutchinson [3] noted that experimental results showed that at the micron scale, smaller metallic material components become stronger during inhomogeneous plastic flows. These are known as size effects, and cannot be observed using the classical plasticity framework, except if one introduces within the yield criterion terms relating to the gradient of the plastic strain [1], [2], [4], [5], [6]. The Aifantis theory has been studied and shown to be thermodynamically consistent in the sense that it obeys the first and second law of thermodynamics [7], [8]. The non-local flow rule obtained in the Aifantis theory is said to be a microscopic force balance augmented by thermodynamically consistent constitutitve relations for the forces involved [8], [9]. Other types of gradient plasticity models developed over the years are the Fleck-Hutchinson [10], [11], [12] and Gao-Nix-Huang strain gradient theories [13], [14]. The reviews of Aifantis [15], [16] provide updates on classes of gradient theories.

Another strain gradient theory worthy of consideration is the Gurtin-Anand theory [17], which makes use of the virtual power principle and the second law of thermodynamics to obtain microscopic force balances and constitutive relations for microscopic stresses. This approach was also used by Borokinni et al. [18] to determine the effects of divergence of plastic strain in the gradient theory of plasticity. The Gurtin-Anand theory established a non-local flow rule -without an assumption of codirectionality constraint - in the form of a tensorial partial differential equation in the plastic strain tensor with accompanying boundary conditions. Whereas the Aifantis flow rule [1] is a scalar partial differential equation - with the assumption of codirectionality - in the accumulated plastic strain. Thus, for this reason, it is said that the Gurtin-Anand theory is richer than the Aifantis theory [7].

However, besides the codirectionality constraint, the tensorial form of the Gurtin-Anand flow rule does not provide a sufficient reason for the major or specific differences between the Gurtin-Anand and Aifantis theories. This is because, as shown by Gurtin et al. [7] the scalar microscopic stresses in the Aifantis theory are resolved components of the elastic and plastic stress tensors. Thus, the Aifantis flow rule can be transformed into a tensorial form involving stress tensors. Working with the fact that the Gurtin-Anand theory is richer in construct than the Aifantis theory, this article uses the Gurtin-Anand theory as a basis to establish the differences between the two theories, and extend the Aifantis theory to accommodate scalar dissipative hardening. 


\subsection{Notations}

In component form, a second-order tensor $\mathbf{A}$ is written as $A_{i j}$ for $i, j=1,2,3$. The expression $\mathbf{A u}$ is written in component form as $A_{i j} u_{j}$, where the summation convention is adopted. The trace of a second-order tensor $\mathbf{A}$ is denoted as $\operatorname{tr} \mathbf{A}$ and written as the sum of the diagonal elements for the matrix of $\mathbf{A}$. The symmetric and skew parts of a second-order tensor $\mathbf{A}$ would be denoted by symA and skwA, respectively, and are defined as $\operatorname{sym} \mathbf{A}=\frac{1}{2}\left(\mathbf{A}+\mathbf{A}^{T}\right)$, skwA $=\frac{1}{2}\left(\mathbf{A}-\mathbf{A}^{T}\right)$, where $\mathbf{A}^{T}$ is the transpose of $\mathbf{A}$. The magnitude of $\mathbf{A}$ is denoted as $|\mathbf{A}|$, and defined as $|\mathbf{A}|=\left(A_{i j} A_{i j}\right)^{1 / 2}$. We define the inner-product ' $:$ ' of nonzero second-order tensors $\mathbf{A}$ and $\mathbf{B}$ by $\mathbf{A}: \mathbf{B}=A_{i j} B_{i j}$. Also, the inner product " ' of the third order tensors $\mathbb{A}$ and $\mathbb{B}$ is denoted by $\mathbb{A} \mathbb{B}$ and defined as $\mathbb{A} \mathbb{B}=A_{i j k} B_{i j k}$. The deviatoric part of a second-order tensor $\mathbf{A}$ would be denoted as $\mathbf{A}_{o}$ or dev $\mathbf{A}$, and defined as $\mathbf{A}_{o}=\operatorname{dev} \mathbf{A}=\mathbf{A}-\frac{1}{3}(\operatorname{tr} \mathbf{A}) \mathbf{I}$, where $\mathbf{I}$ is the second-order unit tensor. Given any nonzero vector $\mathbf{a}$, we define a second-order tensor $(\mathbf{a} \times)$ in component form as $(\mathbf{a} \times)_{i j}=\epsilon_{i k j} a_{k}$, where $\epsilon_{i k j}$ is the permutation symbol.

The partial derivative with respect to the spatial variable would be denoted as $(\cdot)_{, i}$, and defined as $(\cdot)_{{ }_{i}}=\frac{\partial(\cdot)}{\partial x_{i}}$, where $x_{i}$ is the spatial coordinate for $i=1,2,3$. We denote the gradient of a vector field $\mathbf{a}$ and tensor field $\mathbf{A}$ as $\nabla \mathbf{a}$ and $\nabla \mathbf{A}$, respectively, and these are defined in component forms as $(\nabla \mathbf{a})_{i j}=a_{i, j}$ and $(\nabla \mathbf{A})_{i j k}=A_{i j, k}$. The divergence of $\mathbf{a}$ and $\mathbf{A}$ are denoted by diva and $\operatorname{div} \mathbf{A}$, respectively, and are defined in component forms as diva $=a_{k, k}$ and $(\operatorname{DivA})_{i}=A_{i k, k}$. The curl of a tensor $\mathbf{A}$ would be written as CurlA or $\nabla \times \mathbf{A}$ and defined in component form as $(\operatorname{CurlA})_{i j}=\epsilon_{i p q} A_{j q, p}$.

\section{Kinematic relations}

Let $\mathbf{u}(\mathbf{x}, t)$ denote the displacement at a point $\mathbf{x}$ in a body $B$ occupying a region of space. The displacement gradient for small deformation isotropic plastic solids admits the additive decomposition

$$
\nabla \mathbf{u}=\mathbf{H}^{e}+\mathbf{H}^{p} \text { with } \operatorname{tr} \mathbf{H}^{p}=0
$$

into elastic distortion $\mathbf{H}^{e}$ and plastic distortion $\mathbf{H}^{p}$. The tensor $\mathbf{H}^{e}$ characterizes stretching and rotation of the material structure, and can be written as the sum of the lattice strain $\mathbf{E}^{e}$ and lattice rotation $\mathbf{W}^{e}$ which are symmetric and skew-symmetric tensors, respectively. The tensor $\mathbf{H}^{p}$ which is called plastic distortion, arises due to the motion of the dislocation through the material structure. $\mathbf{H}^{p}$ can also be written as the sum of the plastic strain $\mathbf{E}^{p}$ and the plastic rotation $\mathbf{W}^{p}$ which are symmetric and skew-symmetric tensors, respectively. Basic to the works of Aifantis [1] and Gurtin and Anand [17] is the assumption that the plastic rotation $\mathbf{W}^{p}$ is zero. Thus, the basic kinematic rate relation following eq. (1) and the assumption of plastic irrotationality is given by

$$
\nabla \dot{\mathbf{u}}=\dot{\mathbf{H}}^{e}+\dot{\mathbf{E}}^{p} \text { with } \operatorname{tr} \dot{\mathbf{E}}^{p}=0
$$

The flow direction $\mathbf{N}^{p}$ and accumulated plastic strain $e^{p}$ are defined through

$$
\begin{gathered}
\mathbf{N}^{p}=\frac{\dot{\mathbf{E}}^{p}}{\left|\dot{\mathbf{E}}^{p}\right|} \text { for } \dot{\mathbf{E}}^{p} \neq \mathbf{0} \\
\dot{e}^{p}(\mathbf{x}, t)=\left|\dot{\mathbf{E}}^{p}(\mathbf{x}, t)\right| \text { with } e^{p}(\mathbf{x}, 0)=0 .
\end{gathered}
$$

It is clear from eqs. (3) and (4) that

$$
\dot{\mathbf{E}}^{p}=\dot{e}^{p} \mathbf{N}^{p}
$$

Clearly, using eq. (5), the basic rate-like kinematic relation (2) can be written as

$$
\nabla \dot{\mathbf{u}}=\dot{\mathbf{H}}^{e}+\dot{e}^{p} \mathbf{N}^{p}
$$




\section{Macroscopic and microscopic force balances}

Given an arbitrary sub-region $P \subset B$ whose boundary is $\partial P$ with an outward unit normal $\mathbf{n}$ at a point on $\partial P$. In view of the fact that the basic kinematic variables are displacement $\mathbf{u}$, elastic distortion $\mathbf{H}^{e}$ and plastic strain $\mathbf{E}^{p}$, Gurtin and Anand [17] established a gradient theory based on the assumption that there exist

- elastic stress (symmetric) $\mathbf{T}$ power conjugate to the elastic distortion rate $\dot{\mathbf{H}}^{e}$

- plastic microstress $\mathbf{T}^{p}$ power conjugate to the plastic strain rate $\dot{\mathbf{E}}^{p}$

- third-order polar microstress $\mathbb{K}^{p}$ power conjugate to the rate of plastic strain gradient $\nabla \dot{\mathbf{E}}^{p}$

- microtraction $\mathbf{K}(\mathbf{n})$ power conjugate to the plastic strain rate $\dot{\mathbf{E}}^{p}$

- body force $\mathbf{b}$ and macrotraction $\mathbf{t}(\mathbf{n})$ both power conjugate to the velocity $\dot{\mathbf{u}}$.

Given that $\dot{\mathbf{H}}^{e}, \dot{\mathbf{E}}^{p}$ and $\dot{\mathbf{u}}$ are virtual fields consistent with eq. (2), the principle of virtual power states that

$$
\int_{\partial P}\left(\mathbf{t}(\mathbf{n}) \cdot \dot{\mathbf{u}}+\mathbf{K}(\mathbf{n}): \dot{\mathbf{E}}^{p}\right) d A+\int_{P} \mathbf{b} \cdot \dot{\mathbf{u}} d V=\int_{P}\left(\mathbf{T}: \dot{\mathbf{E}}^{e}+\mathbf{T}^{p}: \dot{\mathbf{E}}^{p}+\mathbb{K}^{p}: \nabla \dot{\mathbf{E}}^{p}\right) d V .
$$

The consequences of the virtual power balance are

- Macroscopic force balance and macrotraction condition:

$$
\operatorname{div} \mathbf{T}+\mathbf{b}=0 \text { with } \mathbf{T n}=\mathbf{t}(\mathbf{n}) .
$$

- Microscopic force balance and microtraction condition:

$$
\mathbf{T}_{o}=\mathbf{T}^{p}-\operatorname{div} \mathbb{K}^{p} \text { with } \mathbb{K}^{p}=\mathbf{K}(\mathbf{n}),
$$

where $\mathbf{T}_{o}$ is the deviatortic part of the elastic stress tensor $\mathbf{T}$.

\section{Free-energy imbalance, constitutive relations and flow rule of the Gurtin-Anand gradient theory}

\subsection{Free-energy imbalance}

Let $\psi$ denote the free-energy per unit volume within a subregion $P$ of the body $B$. The second law of thermodynamics asserts that the temporal increase in the global free-energy over the region $P$ cannot exceed the power expended on $P$. A consequence of this law and the power balance is the local free-energy imbalance

$$
\dot{\psi}-\mathbf{T}: \dot{\mathbf{E}}^{e}-\mathbf{T}^{p}: \dot{\mathbf{E}}^{p}-\mathbb{K}^{p}: \nabla \dot{\mathbf{E}}^{p} \leq 0
$$

\subsection{Constitutive relations}

The free-energy $\psi$ is assumed to be function of elastic strain $\mathbf{E}^{e}$ and the Burgers tensor $\mathbf{G}=\mathbf{C}$ url $\mathbf{E}^{p}$ and takes the separable form

$$
\psi=\hat{\psi}^{e}\left(\mathbf{E}^{e}\right)+\hat{\psi}^{p}(\mathbf{G})
$$

into elastic energy $\psi^{e}$ and defect energy $\psi^{p}$. By substitution into eq. (10), we have

$$
\left(\frac{\partial \hat{\psi}^{e}\left(\mathbf{E}^{e}\right)}{\partial \mathbf{E}^{e}}-\mathbf{T}\right): \dot{\mathbf{E}}^{e}-\mathbf{T}^{p}: \dot{\mathbf{E}}^{p}+\left(\mathbb{A}-\mathbb{K}^{p}\right) \vdots \nabla \dot{\mathbf{E}}^{p} \leq 0
$$


where $\mathbb{A}$ can be written in component form

$$
A_{j q p}=\frac{\partial \psi^{p}}{\partial G_{i j}} \epsilon_{i p q} .
$$

Assume that $\mathbb{K}^{p}$ admits additive decomposition

$$
\mathbb{K}^{p}=\mathbb{K}_{e n}^{p}+_{d i s}^{p}
$$

into energetic part $\mathbb{K}_{e n}^{p}$ and dissipative part $\mathbb{K}_{d i s}^{p}$. The energetic polar microstress $\mathbb{K}_{e n}^{p}$ is defined in component form as

$$
\left(\mathbb{K}_{e n}^{p}\right)_{i j k}=\frac{1}{2}\left[A_{i j k}+A_{j i k}\right]-\frac{\delta_{i j}}{3} A_{r r k} .
$$

The free-energy imbalance reduces to the dissipation inequality

$$
\mathbf{T}^{p}: \dot{\mathbf{E}}^{p}+\mathbb{K}_{d i s}^{p} \vdots \dot{\mathbf{E}}^{p} \geq 0 .
$$

Consistent with the dissipation inequality, the constitutive relation for the microscopic stresses are assumed to be [17]

$$
\mathbf{T}^{p}=g\left(d^{p}\right) Y(S) \frac{\dot{\mathbf{E}}^{p}}{d^{p}} \text { and } \mathbb{K}_{d i s}^{p}=l^{2} g\left(d^{p}\right) Y(S) \frac{\nabla \dot{\mathbf{E}}^{p}}{d^{p}}
$$

where $g(\cdot)$ is called rate-sensitivity function, $Y(\cdot)$ is the flow resistance, $S$ is an internal state variable which evolves according to the differential equation

$$
\dot{S}=h(S) d^{p} \text { with } S(\mathbf{x}, 0)=S_{0} .
$$

$d^{p}$ is called effective flow rate defined as

$$
d^{p}=\sqrt{\left|\dot{\mathbf{E}}^{p}\right|^{2}+l^{2}\left|\nabla \dot{\mathbf{E}}^{p}\right|^{2}}
$$

$l$ is called dissipative length scale associated with the microscopic stress $\mathbb{K}_{d i s^{\prime}}^{p} h(\cdot)$ is a hardening function and $S_{0}$ is the initial flow resistance.

\subsection{Gurtin-Anand Flow rule}

The flow rule of viscoplasticity of the Gurtin-Anand type is obtained by augmenting the microscopic force balance eq. (9) with the constitutive relations eq. (14). The flow rule is given as

$$
\mathbf{T}_{0}+\operatorname{div} \hat{\mathbb{K}}_{e n}^{p}(\mathbf{G})=g\left(d^{p}\right) Y(S) \frac{\dot{\mathbf{E}}^{p}}{d^{p}}-\operatorname{div}\left(l^{2} g\left(d^{p}\right) Y(S) \frac{\nabla \dot{\mathbf{E}}^{p}}{d^{p}}\right)
$$

where $\hat{\mathbb{K}}_{e n}^{p}(\mathbf{G})$ is a constitutive response function for the energetic microscopic stress $\mathbb{K}_{e n}^{p}$ and the term $-\operatorname{div} \widehat{\mathbb{K}}_{e n}^{p}(\mathbf{G})$ is called the backstress which accounts for kinematic hardening. The terms on the right hand side of eq. (17) account for dissipative hardening.

\subsubsection{Remark}

A quadratic and isotropic form of the defect energy $\psi^{p}$ is [17] 


$$
\psi^{p}(\mathbf{G})=\frac{1}{2} \mu L^{2}|\mathbf{G}|^{2},
$$

which when substituted into the flow rule is

$$
\mathbf{T}_{o}+\mu L^{2}\left(\Delta \mathbf{E}^{p}-\operatorname{sym}_{o}\left(\nabla \operatorname{div} \mathbf{E}^{p}\right)\right)=g\left(d^{p}\right) Y(S) \frac{\dot{\mathbf{E}}^{p}}{d^{p}}-\operatorname{div}\left(l^{2} g\left(d^{p}\right) Y(S) \frac{\nabla \dot{\mathbf{E}}^{p}}{d^{p}}\right),
$$

where $\operatorname{sym}_{o} \mathbf{V}$ is called symmetric and deviatoric part of a tensor $\mathbf{V}$ defined by

$$
\operatorname{sym}_{o} \mathbf{V}=\frac{1}{2}\left(\mathbf{V}+\mathbf{V}^{T}\right)-\frac{1}{3}(\operatorname{tr} \mathbf{V}) \mathbf{I}
$$

\section{The Aifantis-based theory and the Gurtin-Anand Model}

The Aifantis theory assumes from the outset that the codirectionality hypothesis is satisfied. This means that the deviatoric stress $\mathbf{T}_{o}$ and flow direction $\mathbf{N}^{p}$ are in the same direction. Two constraints are needed for which the Aifantis-based theory is a special case of the Gurtin-Anand theory. The first constraint has been identified as the codirectionality constraint. The second constraint which will be shown later, states that the gradient of the flow direction is zero.

\subsection{Microscopic force balance of the Aifantis-based theory}

Assume that the codirectionality hypothesis holds. Consider the microscopic force balance of the Gurtin-Anand model given by eq. (9). The resolved component of the elastic stress $\mathbf{T}_{0}$ to the flow direction would be denoted by $\tau$ and resolved component of the plastic stress $\mathbf{T}^{p}$ to the flow direction would be denoted as $\tau^{p}$ so that

$$
\tau=\mathbf{T}_{o}: \mathbf{N}^{p} \text {, and } \tau^{p}=\mathbf{T}^{p}: \mathbf{N}^{p} .
$$

We define the operation ' $\otimes$ ' on the pair (A, a) (with $\mathbf{A}$ a second tensor and $\mathbf{a}$ a vector) by

$$
(\mathbf{A} \otimes \mathbf{a})_{i j k}=A_{i j} a_{k} .
$$

It is easy to show that given any nonzero vector a, then

$$
\operatorname{div} \mathbf{a}=\mathbf{N}^{p}: \operatorname{div}\left(\mathbf{N}^{p} \otimes \mathbf{a}\right) .
$$

To show this, recall that $\left|\mathbf{N}^{p}\right|=1$ and that $N_{i j, k}^{p} N_{i j}^{p}=0$. Clearly,

$$
\mathbf{N}^{p}: \operatorname{div}\left(\mathbf{N}^{p} \otimes \mathbf{a}\right)=N_{i j}^{p}\left(N_{i j}^{p} a_{k}\right)_{, k}=a_{k, k}=\operatorname{diva} .
$$

Resolving the Gurtin-Anand microscopic force balance along the flow direction, we have

$$
\tau=\tau^{p}-\mathbf{N}^{p}: \operatorname{div} \mathbb{K}^{p}
$$

The constitutive relation for the microscopic stress $\mathbf{T}^{p}$ [see eq. (14) $)_{1}$ shows that $\mathbf{T}^{p}$ and $\mathbf{N}^{p}$ are in the same direction. Using the Gurtin-Anand microscopic force balance, it is clear that $\operatorname{div} \mathbb{K}^{p}$ is in the direction of $\mathbf{N}^{p}$.

Consider the power $\mathbb{K}^{p} \vdots \nabla \dot{\mathbf{E}}^{p}$ expended within an arbitrary portion of the body by the microscopic stress $\mathbb{K}^{p}$. In terms of the accumulated plastic strain rate we have 


$$
\mathbb{K}^{p} \vdots \nabla \dot{\mathbf{E}}^{p}=\left(\mathbb{K}^{p} \vdots \nabla \mathbf{N}^{p}\right) \dot{e}^{p}+\left(\mathbb{K}^{p} \mathbf{N}^{p}\right) \cdot \nabla \dot{e}^{p}
$$

where we define $\mathbb{K}^{p} \mathbf{N}^{p}$ in the component form as

$$
\left(\mathbb{K}^{p} \mathbf{N}^{p}\right)_{k}=K_{i j k}^{p} N_{i j}^{p} .
$$

If

$$
\vec{\xi}^{p}=\mathbb{K}^{p} \mathbf{N}^{p} \text { and } \phi^{p}=\mathbb{K}^{p} \vdots \nabla \mathbf{N}^{p},
$$

then we have

$$
\mathbb{K}^{p}: \nabla \dot{\mathbf{E}}^{p}=\phi^{p} \dot{e}^{p}+\vec{\xi}^{p} \cdot \nabla \dot{e}^{p} .
$$

Basic to obtaining the Aifantis flow rule is the assumption that $\phi^{p}=0$, so that we have

$$
\mathbb{K}^{p} \vdots \nabla \dot{\mathbf{E}}^{p}=\vec{\xi}^{p} \cdot \nabla \dot{e}^{p}
$$

It is clear from eq. (26) that

$$
\mathbb{K}^{p}=\mathbf{N}^{p} \otimes \vec{\xi}^{p},
$$

Hence, following eq. (23), the Aifantis microscopic force balance is given by

$$
\tau=\tau^{p}-\operatorname{div} \vec{\xi}^{p} \text {, where } \mathbb{K}^{p}=\mathbf{N}^{p} \otimes \vec{\xi}^{p}
$$

\subsubsection{Motivation and jusfication for the choice of eq. (26)}

The gradient $\nabla \dot{e}^{p}$ of the rate of accumulated plastic strain can be written as

$$
\left(\nabla\left|\dot{\mathbf{E}}^{p}\right|\right)_{k}=\left[\left(\dot{E}_{i j}^{p} \dot{E}_{i j}^{p}\right)^{1 / 2}\right]_{, k}=\dot{E}_{i j, k}^{p} N_{i j}^{p}
$$

so that there exists polar microscopic stress

$$
\mathbb{K}^{p *}=\mathbf{N}^{p} \otimes \vec{\xi}^{* p}
$$

such that

$$
\vec{\xi}^{* p} \cdot \nabla \dot{e}^{p}=\xi_{k}^{* p} N_{i j}^{p} \dot{E}_{i j, k}^{p}=\mathbb{K}^{p *} \vdots \nabla \dot{\mathbf{E}}^{p} .
$$

\subsubsection{Remark}

The transformation of the Gurtin-Anand type microscopic force balance to the Aifantis type microscopic force balance shows that the Aifantis model can account for backstress, and we can formulate a constitutive theory based on the Aifantis model that accommodates the Burgers tensor as an independent variable of the defect energy.

Two constraints are identified for which the Aifantis theory is a special case of the Gurtin-Anand theory. These are

- Codirectionality hypothesis

- the polar microscopic stress $\mathbb{K}^{p}$ and the gradient of the flow direction $\nabla \mathbf{N}^{p}$ are orthogonal in the sense $\mathbb{K}^{p} \nabla \mathbf{N}^{p}$ $=0$. To be more specific, we shall show later that $\nabla \mathbf{N}^{p}=0$. 


\section{Free-energy imbalance of the Aifantis based theory}

Using the Gurtin-Anand free-energy imbalance and eqs. (20) and (26), the free-energy imbalance for the Aifantis based theory is given by

$$
\dot{\psi}-\mathbf{T}: \dot{\mathbf{E}}^{e}-\tau^{p} \dot{e}^{p}-\vec{\xi}^{p} \cdot \nabla \dot{e}^{p} \leq 0 .
$$

This inequality implies that the constitutive relations for the microscopic stresses $\tau^{p}$ and $\vec{\xi}^{p}$ can be obtained from the constitutive relations for $\mathbf{T}^{p}$ and $\mathbb{K}^{p}$ by resolving the microscopic stresses $\mathbf{T}^{p}$ and $\mathbb{K}^{p}$ on the flow direction $\mathbf{N}^{p}$.

\section{Constitutive relations for the Aifantis-based theory}

\subsection{Constitutive relations for energetic microstresses}

We recall that the free-energey admits decomposition into elastic free energy $\psi^{e}$ and defect energy $\psi^{p}$ via

$$
\psi=\hat{\psi}^{e}\left(\mathbf{E}^{e}\right)+\psi^{p}(\mathbf{G}),
$$

where $\mathbf{G}=$ curlE $^{p}$. The standard result shows that

$$
\mathbf{T}=\frac{\partial \hat{\psi}^{e}\left(\mathbf{E}^{e}\right)}{\partial \mathbf{E}^{e}} .
$$

Following Borokinni and Ajayi [9], the Burgers tensor rate can be written as

$$
\dot{\mathbf{G}}=\left(\nabla \dot{e}^{p} \times\right) \mathbf{N}^{p}+\dot{e}^{p}\left(\operatorname{curl} \mathbf{N}^{p}\right),
$$

where $\left(\nabla \dot{e}^{p} \times\right)$ in component form is written as $\left(\nabla \dot{e}^{p} \times\right)_{i j}=\epsilon_{i k j} \dot{e}_{, k}^{p}$.

Basic to what follows, we assume a simple constitutive kinematic relation for $\mathbf{G}$ through

$$
\mathbf{G}=\hat{\mathbf{G}}\left(e^{p}, \nabla e^{p}\right),
$$

so that $\dot{\mathbf{G}}$ can be written in component form as

$$
\dot{G}_{i j}=\frac{\partial \hat{G}_{i j}}{\partial e_{, k}^{p}} \dot{e}_{, k}^{p}+\frac{\partial \hat{G}_{i j}}{\partial e^{p}} \dot{e}^{p} .
$$

By comparing eq. (36) with eq. (34), it is clear that

$$
\frac{\partial \mathbf{G}}{\partial e^{p}}=\operatorname{curl} \mathbf{N}^{p}, \text { and } \frac{\partial G_{i r}}{\partial e_{, k}^{p}}=N_{j r}^{p} \epsilon_{i k j} .
$$

The defect energy can be written as function of $\mathbf{G}$ or as function of $e^{p}$ and $\nabla e^{p}$. Clearly,

$$
\psi^{p}=\hat{\psi}^{p}(\mathbf{G})=\psi^{p *}\left(e^{p}, \nabla e^{p}\right),
$$

and we write

$$
\dot{\psi}^{p}=\frac{\partial \hat{\psi}^{p}(\mathbf{G})}{\partial \mathbf{G}}: \dot{\mathbf{G}}=\frac{\partial \psi^{p *}}{\partial e^{p}} \dot{e}^{p}+\frac{\partial \psi^{p *}}{\partial \nabla e^{p}} \cdot \nabla \dot{e}^{p}
$$


By eq. (37), we have

$$
\frac{\partial \psi^{p *}}{\partial e^{p}}=\frac{\partial \hat{\psi}^{p}}{\partial \mathbf{G}}: \operatorname{curl} \mathbf{N}^{p} \text {, and }\left(\frac{\partial \psi^{p *}}{\partial \nabla e^{p}}\right)_{k}=\frac{\partial \hat{\psi}}{\partial G_{i r}} N_{j r}^{p} \epsilon_{i k j} .
$$

The Aifantis based theory considers the derivatives $\frac{\partial \psi^{p *}}{\partial e^{p}}$ and $\frac{\partial \psi^{p *}}{\partial \nabla e^{p}}$ as energetic microscopic stresses of $\tau^{p}$ and $\vec{\xi}^{p}$, respectively, and we denote these as $\tau_{e n}^{p}$ and $\vec{\xi}_{e n}^{p}$, i.e.

$$
\tau_{e n}^{p}=\frac{\partial \psi^{p *}}{\partial e^{p}}, \text { and } \vec{\xi}_{e n}^{p}=\frac{\partial \psi^{p *}}{\partial \nabla e^{p}}
$$

From the mechanics of the problem being considered, the microscopic scalar stress $\tau^{p}$ is purely dissipative so that $\tau_{e n}^{p}=0$ and hence the defect energy $\psi^{p^{*}}$ is independent of the accumulation of the plastic strain. A consequence of this is that the quantities $\frac{\partial \hat{\psi}^{p}}{\partial \mathbf{G}}$ and $\frac{\partial \mathbf{G}}{\partial e^{p}}$ are orthogonal. The dissipative microscopic stress $\vec{\xi}_{d i s}^{p}$ is defined by

$$
\vec{\xi}_{\text {dis }}^{p}=\vec{\xi}^{p}-\vec{\xi}_{e n}^{p}
$$

By substituting eqs. (33) and (40) in the free-energy imbalance we have reduced dissipation inequality

$$
\tau^{p} \dot{e}^{p}+\vec{\xi}_{d i s}^{p} \cdot \nabla \dot{e}^{p} \geq 0
$$

It can be recalled that the temporal increase in the defect energy can be written as

$$
\dot{\psi}^{p}=\mathbb{K}_{e n}^{p}: \nabla \dot{\mathbf{E}}^{p}
$$

By eq. (40) and the fact that the defect energy $\hat{\psi}^{p}$ is independent of accumulation of plastic strain, we have

$$
\mathbb{K}_{e n}^{p}: \nabla \dot{\mathbf{E}}^{p}=\vec{\xi}_{e n}^{p} \cdot \nabla \dot{e}^{p}
$$

It is clear from eqs. (29) and (30) that

$$
\mathbb{K}_{e n}^{p}=\mathbf{N}^{p} \otimes \vec{\xi}_{e n}^{p}=\mathbf{N}^{p} \otimes \frac{\partial \hat{\psi}^{p}}{\partial \nabla e^{p}}
$$

\section{The Aifantis flow rule obtained from the Gurtin-Anand strain gradient theory}

Using eqs. (27), (41) and (44), the dissipative polar microscopic stress $\mathbb{K}_{\text {dis }}^{p}$ is given

$$
\mathbb{K}_{d i s}^{p}=\mathbf{N}^{p} \otimes \vec{\xi}_{d i s^{\prime}}^{p}, \text { where } \vec{\xi}_{d i s}^{p}=\hat{\vec{\xi}}_{d i s}^{p}\left(\mathbf{N}^{p}, \dot{e}^{p}, \nabla \dot{e}^{p}\right)
$$

In the Aifantis flow rule, there are no contribution from the dissipative microstress vector $\vec{\xi}_{\text {dis }}^{p}$ so that $\mathbb{K}_{d i s}^{p}=\mathbf{0}$. by

The Aifantis microscopic force balance obtained from the Gurtin-Anand microscopic force balance is given

$$
\mathbf{T}_{o}: \mathbf{N}^{p}=\mathbf{T}^{p}: \mathbf{N}^{p}-\mathbf{N}^{p}: \operatorname{div} \mathbb{K}_{e n}^{p}
$$

Using eq. (3) and the constitutive relation (14) and noting the the effective flow rate reduces to $d^{p}=\left|\dot{\mathbf{E}}^{p}\right|=\dot{e}^{p}$ we have 
and by eq. (22)

$$
\mathbf{N}^{p}: \operatorname{div} \mathbb{K}_{e n}^{p}=\mathbf{N}^{p}: \operatorname{div}\left(\mathbf{N}^{p} \otimes \frac{\partial \psi^{p *}}{\partial \nabla e^{p}}\right)=\operatorname{div}\left(\frac{\partial \psi^{p *}}{\partial \nabla e^{p}}\right) .
$$

By substituting eqs. (47) and (48) in (23) we have the flow rule given as

$$
\tau=Y(S)-\operatorname{div}\left(\frac{\partial \psi^{p *}}{\partial \nabla e^{p}}\right)
$$

\subsection{Quadratic defect energy}

Assume that the free energies $\hat{\psi}^{p}(\mathbf{G})$ and $\psi^{p^{*}}\left(\nabla e^{p}\right)$ take the isotropic and quadratic form

$$
\psi^{p *}\left(\nabla e^{p}\right)=\frac{1}{2} \beta\left|\nabla e^{p}\right|^{2}, \text { with } \hat{\psi}^{p}(\mathbf{G})=\psi^{p *}\left(\nabla e^{p}\right) .
$$

Using eqs. (41) and (50) it is clear that

$$
\mathbb{K}_{e n}^{p}=\beta \mathbf{N}^{p} \otimes \nabla e^{p}
$$

The Aifantis flow rule (49) for the quadratic defect energy (50) is obtained as

$$
\tau=Y\left(e^{p}\right)-\beta \Delta e^{p}
$$

\subsubsection{Remark}

The term $-\beta \Delta e^{p}$ when compared with the term $\operatorname{div} \mathbb{K}_{e n}^{p}$ in the Gurtin-Anand flow rule measures backstress, so that the Aifantis flow rule also accounts for the Bauschinger effect.

\section{Generalized Aifantis-based theory}

The Gurtin-Anand microscopic force balance can be written as

$$
\mathbf{T}_{o}=\mathbf{T}^{p}-\operatorname{div} \mathbb{K}_{e n}^{p}-\operatorname{div} \mathbb{K}_{d i s^{\prime}}^{p}
$$

where

$$
\mathbf{T}^{p}=g\left(d^{p}\right) Y(S) \frac{\dot{\mathbf{E}}^{p}}{d^{p}} \text { and } \mathbb{K}_{d i s}^{p}=l^{2} g\left(d^{p}\right) Y(S) \frac{\nabla \dot{\mathbf{E}}^{p}}{d^{p}}
$$

Recall that $\mathbb{K}_{d i s}^{p}=\mathbf{N}^{p} \otimes \vec{\xi}_{d i s}^{p}$, so that

$$
\operatorname{div} \vec{\zeta}_{d i s}^{p}=\mathbf{N}^{p}: \operatorname{div} \mathbb{K}_{d i s}^{p}
$$


Using the identity $N_{i j k}^{p} N_{i j}^{p}=0$, it is clear that

$$
\mathbb{K}_{\text {en }}^{p} \vdots \nabla \mathbf{N}^{p}=0 \text { and } \mathbb{K}_{\text {dis }}^{p} \vdots \nabla \mathbf{N}^{p}=0
$$

Using eqs. (55) we have

$$
\mathbb{K}_{d i s}^{p} \vdots \nabla \mathbf{N}^{p}=l^{2} \frac{g\left(d^{p}\right) Y(S)}{d^{p}}\left(\nabla \dot{\mathbf{E}}^{p}\right) \vdots \nabla \mathbf{N}^{p}=l^{2} \frac{g\left(d^{p}\right) Y(S)}{d^{p}}\left|\nabla \mathbf{N}^{p}\right|^{2} \dot{e}^{p}=0
$$

This implies that

$$
\nabla \mathbf{N}^{p}=\mathbf{0} .
$$

Resolving eq. (53) in the direction of the flow direction $\mathbf{N}^{p}$ we have

$$
\tau=\tau^{p}-\operatorname{div} \vec{\xi}_{e n}^{p}-\operatorname{div} \vec{\xi}_{d i s^{\prime}}^{p}
$$

It is clear that

$$
\tau^{p}=\mathbf{T}^{p}: \mathbf{N}^{p}=g\left(d^{p}\right) Y(S) \frac{\dot{\mathbf{E}}^{p}: \mathbf{N}^{p}}{d^{p}}=g\left(d^{p}\right) Y(S) \frac{\dot{e}^{p}}{d^{p}}
$$

and

$$
\left(\mathbb{K}_{d i s}^{p}\right)_{i j k}=l^{2} g\left(d^{p}\right) Y(S)\left(\frac{\dot{e}^{p},{ }_{k} N_{i j}^{p}+N_{i j, k}^{p} \dot{e}^{p}}{d^{p}}\right)
$$

so that by eqs. (54) and (56), we have

$$
\left(\vec{\xi}_{d i s}^{p}\right)_{k}=N_{i j}^{p}\left(\mathbb{K}_{d i s}^{p}\right)_{i j k}=l^{2} g\left(d^{p}\right) Y(S) \frac{e^{p}, k}{d^{p}}
$$

The effective flow rate can be written in terms of the accumlated plastic strain and the flow direction as

$$
d^{p}=\sqrt{\left|\dot{\mathbf{E}}^{p}\right|^{2}+l^{2}\left|\nabla \dot{\mathbf{E}}^{p}\right|^{2}}=\sqrt{\left(1+l^{2}\left|\nabla \mathbf{N}^{p}\right|^{2}\right)\left|\dot{e}^{p}\right|^{2}+l^{2}\left|\nabla \dot{e}^{p}\right|^{2}} \text { with } \mathbf{N}^{p}=\frac{\mathbf{T}_{o}}{\left|\mathbf{T}_{o}\right|} .
$$

By eq. (56), we have

$$
d^{p}=\sqrt{\left|\dot{e}^{p}\right|^{2}+l^{2}\left|\nabla \dot{e}^{p}\right|^{2}}
$$

Using the quadratic defect energy (50) and substituting eqs. (58) and (59) in (57) we have a generalized Aifantisbased flow rule given as

$$
\tau+\beta \Delta e^{p}=g\left(d^{p}\right) Y(S) \frac{\dot{e}^{p}}{d^{p}}-\operatorname{div}\left(l^{2} g\left(d^{p}\right) Y(S) \frac{\nabla \dot{e}^{p}}{d^{p}}\right)
$$

\section{Conclusion}

Eq. (52) is the equivalent classical Aifantis flow rule derived from the use of codirectionality constraint and orthogonality conditions of polar microscopic stress and gradient of flow direction. However, in deriving the generalized Aifantis-based flow rule [Eq. (61)], it is obtained that the gradient of flow direction is null. As observed in the last term of Eq. (61), the generalized Aifantis-based flow rule accounts for scalar dissipative hardening. The Gurtin-Anand flow rule is richer than the Aifantis flow rule, and the main differences are observed from the fact that the Aifantis flow rule will be recovered from the Gurtin-Anand flow rule whenever codirectionality constraint is imposed and the gradient of flow direction is zero. 


\section{References}

[1] Aifantis EC. Trans. ASME]. Eng. Mater. Tech. 1984, 106, 326-330.

[2] Aifantis EC. Int. J. Plast. 1987, 3, 211-247.

[3] Hutchinson JW. Int. J. Solids Struct. 2000, 37, 225-238.

[4] Muhlhaus HB, Aifantis EC. Int. J. Solids Struct. 1991, 28, 845-857.

[5] Aifantis EC, Walgraef D, Zbib HM, eds. Material Instabilities. Special issue of Res Mechanica. Elsevier Applied Science Publisher, 1988, 23, 2-3, 97-305.

[6] Muhlhaus H-B, ed. Continuum Models for Materials with Microstructure. Wiley: Chichester, 1995.

[7] Gurtin ME, Fried E, Anand L. The Mechanics and Thermodynamics of Continua. Cambridge University Press: Cambridge, 2010.

[8] Curtin ME, Anand L. J. Mech. Phys. Solids 2009, 57, 405-421.

[9] Borokinni AS, Ajayi KF. Mech. Res. Comm. 2017, 84, 110-115.

[10] Fleck NA, Hutchinson JW. J. Mech. Phys. Solids. 1993, 41, 1825-1857.

[11] Fleck NA, Hutchinson JW. In: Hutchinson JW, Wu TY, eds. Advances in Applied Mechanics, Vol. 33. Academic Press: New York, 1997, 295-361.

[12] Fleck NA, Muller CM, Ashby MF, Hutchinson JW. Acta. Metall. Mater. 1994, 42, 475-487.

[13] Gao H, Huang Y, Nix WD, Hutchinson JW. J. Mech. Phys. Solids 1999, 47, 1239-1263.

[14] Huang Y, Gao H, Nix WD, Hutchinson JW. J. Mech. Phys. Solids 2000a, 48, 99-128.

[15] Aifantis EC. Mech. Mater. 2003, 35, 259-280.

[16] Aifantis EC. Adv. Appl. Mech. 2016, 49, 1-110.

[17] Gurtin ME, Anand L. J. Mech. Phys. Solids 2005, 53, 1624-1649.

[18] Borokinni A, Akinola A, Layeni O. Theor. Appl. Mech (Belgrade) 2017, 44, 1-13. 